\title{
MicroRNA-497-5p downregulation inhibits cell viability, reduces extracellular matrix deposition and induces apoptosis in human hyperplastic scar fibroblasts by regulating Smad7
}

\author{
ZHIQIANG LI, PENGTAO WANG, JIE ZHANG and DONGKUI ZHAO \\ Shanghai Meizhizhen Medical Cosmetology Clinic, Shanghai 200122, P.R. China
}

Received February 13, 2020; Accepted July 7, 2020

DOI: $10.3892 /$ etm.2021.9815

\begin{abstract}
Hypertrophic scars (HSs) are characterized by excessive extracellular matrix deposition and excessive growth of dense fibrous tissues. MicroRNAs (miRNAs/miRs) serve key roles in HS formation. The present study investigated the expression, role and mechanism underlying the effects of miR-497-5p in HS formation. miR-497-5p expression was detected via reverse transcription-quantitative PCR. The association between miR-497-5p and Smad7 was analyzed using TargetScan and luciferase reporter assays. Protein expression levels of extracellular matrix markers were measured via western blotting. Cell viability and apoptosis were determined using the Cell Counting Kit- 8 assay and flow cytometry, respectively. The results suggested that miR-497-5p expression was upregulated in HS tissues and human HS fibroblasts (hHSFs) compared with healthy control skin tissues and CCC-ESF-1 cells, respectively. Smad7 was directly targeted by miR-497-5p, and was downregulated in HS tissues and hHSFs compared with healthy control skin tissues and CCC-ESF-1 cells, respectively. Moreover, Smad7 upregulation significantly inhibited cell viability, decreased extracellular matrix deposition and induced apoptosis in hHSFs compared with the control-plasmid group. Moreover, the results indicated that, compared with the inhibitor control group, miR-497-5p inhibitor inhibited cell viability, decreased extracellular matrix deposition and induced apoptosis in hHSFs, which were significantly reversed by Smad7 knockdown. In conclusion, the results indicated that $\mathrm{miR}-497-5 \mathrm{p}$ downregulation repressed HS formation by inhibiting extracellular matrix deposition and hHSF proliferation at least partly by targeting Smad7.
\end{abstract}

Correspondence to: Mr. Zhiqiang Li, Shanghai Meizhizhen Medical Cosmetology Clinic, 898 Puming Road, Pudong, Shanghai 200122, P.R. China

E-mail:1111112345@163.com

Key words: hypertrophic scar, extracellular matrix, microR-497-5p, Smad7, fibroblasts

\section{Introduction}

Hypertrophic scars (HS) caused by various traumas are a product of the healing process and seriously affect the patient's physical health (1-3). HS is characterized by hyperproliferation of scar fibroblasts and excessive accumulation of extracellular matrix (ECM) $(4,5)$. At present, the main treatment strategy for HS is surgical excision with superficial X-ray radiation and dot-array laser (6); however, surgical treatment may cause secondary injuries (7), and radiation therapy can cause radiation dermatitis, delayed incision healing and skin canceration at the irradiation site $(6,7)$. Therefore, identifying the molecular mechanisms underlying HS formation to aid with the development of novel therapeutic strategies for HS is important.

MicroRNAs (miRNAs/miRs) are endogenous, non-coding, single-stranded small RNAs, which regulate gene and protein expression at the post-transcriptional level by binding to target genes in a complementary or incompletely complementary manner (8). Evidence has indicated that miRNAs serve key roles in pathophysiological processes, such as cell proliferation, apoptosis, organ development and immune regulation (8-12). Increasing evidence has also suggested that miRNAs serve a critical role in HS formation (13-16). miR-497-5p has been reported to serve critical roles in various types of cancer via regulating cell proliferation and apoptosis, and modulating the expression of several genes and proteins (17-20). In addition, the important roles of miR-497-5p in myofibroblast differentiation and pulmonary fibrogenesis have been reported (21). The aforementioned studies indicated that miR-497-5p may serve an important role in the occurrence and development of HS. However, the role of miR-497-5p in HS formation is not completely understood.

Smad7 serves as a negative regulator of transforming growth factor (TGF)- $\beta$ signaling via multiple mechanisms, such as TGF- $\beta$ type I receptor degradation induction, Smad2/3 phosphorylation and recruitment blocking $(22,23)$. Smad7 can protect against TGF- $\beta$-induced fibrosis in various organs, including the lungs, kidneys and liver $(24,25)$. Several studies have reported an important role of $\mathrm{Smad} 7$ in the regulation of fibroblast proliferation and ECM accumulation (26-28), indicating an important role for Smad7 in HS formation.

The aim of the present study was to investigate the expression and role of miR-497-5p in HS formation, and to further explore the underlying molecular mechanism. 


\section{Materials and methods}

Clinical samples. A total of 22 HS tissues (age 27-59 years; 11 male patients and 11 female patients) were collected during scar excision at Shanghai Meizhen Medical Cosmetology Clinic. Additionally, 22 healthy control skin tissues (age, 24-63 years; 11 male patients and 11 female patients) were collected during auto-skin grafting at Shanghai Meizhen Medical Cosmetology Clinic between May 23, 2017 and June 4, 2019. Written informed consent was obtained from the patients and all patients approved the use of their samples in the present study. The present study was approved by the Ethics Committee of Shanghai Meizhizhen Medical Cosmetology Clinic.

Cell culture and cell transfection. Human embryonic skin fibroblasts CCC-ESF-1 (cat. no. YB-ATCC-3084; Shanghai Zibo Biological Technology Co., Ltd.) and human HS fibroblasts (hHSFs; cat. no. C0618; Shanghai Guandao Biological engineering Co., Ltd.) were cultured in RPMI-1640 medium (Gibco; Thermo Fisher Scientific, Inc.) containing 10\% FBS (Gibco; Thermo Fisher Scientific, Inc.) at $37^{\circ} \mathrm{C}$ with $5 \% \mathrm{CO}_{2}$.

Control-plasmid (1 $\mu \mathrm{g}$; cat. no. sc-437275; Santa Cruz Biotechnology, Inc.), Smad7-plasmid (1 $\mu \mathrm{g}$; cat. no. sc400251-ACT; Santa Cruz Biotechnology, Inc.), miR-497-5p inhibitor (100 nM; 5'-ACAAACCACAGTGTGCTGCTG-3'; Sangon Biotech Co., Ltd.), inhibitor control (100 nM; 5'-CAG TACTTTTGTGTAGTACAA-3'; Sangon Biotech Co., Ltd.), Smad7-short hairpin (sh)RNA (1 $\mu \mathrm{g}$; cat. no. sc-36508-SH; Santa Cruz Biotechnology, Inc.), control-shRNA (1 $\mu \mathrm{g}$; cat. no. sc-108060; Santa Cruz Biotechnology, Inc.) or $100 \mathrm{nM}$ miR-497-5p inhibitor $+1 \mu \mathrm{g}$ Smad7-shRNA were transfected into hHSFs $\left(5 \times 10^{4}\right.$ per well) using Lipofectamine ${ }^{\circledR} 2000$ (Invitrogen; Thermo Fisher Scientific, Inc.) according to the manufacturer's instructions. At $48 \mathrm{~h}$ post-transfection, transfection efficiency was assessed via reverse transcription-quantitative PCR (RT-qPCR).

Luciferase reporter analysis. The binding sites between miR-497-5p and Smad7 were predicted by TargetScan version 7.2 (www.targetscan.org/vert_72). To confirm the binding sites between miR-497-5p and Smad7, a dual-luciferase reporter assay was conducted. Briefly, the wild-type (WT-Smad7) and mutant (MUT-Smad7) 3'-untranslated region (UTR) of Smad7 were cloned into a pmiR-RB-Report ${ }^{\mathrm{TM}}$ dual luciferase reporter gene plasmid vector (Guangzhou RiboBio Co., Ltd.) according to the manufacturer's instructions. hHSFs $\left(5 \times 10^{4}\right.$ per well) were co-transfected with $1 \mathrm{ng}$ WT-Smad7 or $1 \mathrm{ng}$ MUT-Smad7 and $50 \mathrm{nM}$ miR-497-5p mimic (5'-CAGCAGCACACUGUGGUU UGUAAACCACAGUGUGCUGCUGUU-3'; Guangzhou RiboBio Co., Ltd.) or $50 \mathrm{nM}$ mimic control (5'-UUCUCC GAACGUGUCACGUTT-3'; Guangzhou RiboBio Co., Ltd.) using Lipofectamine ${ }^{\circledR} 2000$ (Invitrogen; Thermo Fisher Scientific, Inc.). Renilla luciferase pRL-TK vector (Promega Corporation) was used as the internal control. At $48 \mathrm{~h}$ post-transfection, relative luciferase activities were assessed using the Dual-Luciferase Reporter Assay system (Promega Corporation). Firefly luciferase activity was normalized to Renilla luciferase activity.
Cell Counting Kit-8 (CCK-8) assay. The CCK-8 assay (Beyotime Institute of Biotechnology) was performed to assess hHSF cell viability according to the manufacturer's protocol. hHSFs seeded $\left(1 \times 10^{4}\right.$ cells/well) into 96-well plates were transfected with $1 \mu \mathrm{g}$ control-plasmid, $1 \mu \mathrm{g}$ Smad7-plasmid, $100 \mathrm{nM}$ miR-497-5p inhibitor, $100 \mathrm{nM}$ inhibitor control or $100 \mathrm{nM}$ miR-497-5p inhibitor $+1 \mu \mathrm{g} \mathrm{Smad7-shRNA}$. At $48 \mathrm{~h}$ post-transfection, $10 \mu 1$ CCK- 8 solution was added to each well and incubated at $37^{\circ} \mathrm{C}$ with $5 \% \mathrm{CO}_{2}$ for $4 \mathrm{~h}$. Subsequently, hHSF cell viability was assessed by measuring the absorbance at a wavelength of $490 \mathrm{~nm}$ using a microplate reader.

Flow cytometry assay. At $48 \mathrm{~h}$ post-transfection, hHSF apoptosis was assessed using the Annexin V-FITC/PI apoptosis detection kit (Beyotime Institute of Biotechnology) according to the manufacturer's instructions. FACScan flow cytometer (Beckman Coulter, Inc.) was used to detect early and late hHSF apoptosis. Data were analyzed using FlowJo software version 7.6.1 (FlowJo LLC).

$R T-q P C R$. Total RNA was extracted from tissues or cells using TRIzol $^{\circledR}$ (Invitrogen; Thermo Fisher Scientific, Inc.) according to the manufacturer's instructions. Total RNA was reverse transcribed into cDNA using the miScript Reverse Transcription kit (Qiagen $\mathrm{GmbH}$ ) according to the manufacturer's instructions. The conditions for reverse transcription were as follows: $70^{\circ} \mathrm{C}$ for $5 \mathrm{~min}, 37^{\circ} \mathrm{C}$ for $5 \mathrm{~min}$ and $42^{\circ} \mathrm{C}$ for $60 \mathrm{~min}$. Subsequently, qPCR was performed using the QuantiFast SYBR Green PCR kit (Qiagen GmbH). The following thermocycling conditions were used for qPCR: Initial denaturation at $95^{\circ} \mathrm{C}$ for $10 \mathrm{~min}$; followed by 35 cycles of $15 \mathrm{sec}$ at $95^{\circ} \mathrm{C}$, $40 \mathrm{sec}$ at $55^{\circ} \mathrm{C}$ and a final extension step at $72^{\circ} \mathrm{C}$ for $30 \mathrm{sec}$. miRNA and mRNA expression levels were quantified using the $2^{-\Delta \Delta C q}$ method (29) and normalized to the internal reference genes U6 and GAPDH, respectively. The primer sequences used for PCR were as follows: GAPDH forward, 5'-TGTTGC CATCAATGACCCCTT-3' and reverse, 5'-CTCCACGAC GTACTCAGCG-3'; Smad7 forward, 5'-GCTCCCATCCTG TGTGTTAA-3' and reverse, 5'-TAGGTGTCAGCCTAGGAT GGT-3'; U6 forward, 5'-GCTTCGGCAGCACATATACTA AAAT-3' and reverse, 5'-CGCTTCACGAATTTGCGTGTC AT-3'; miR-497-5p forward, 5'-ATCCAGTGCGTGTCGTG-3' and reverse, 5'-TGCTCAGCAGCACACTGT-3'.

Western blotting. Total protein was extracted from tissues or cells using RIPA lysis buffer (Beyotime Institute of Biotechnology). A bicinchoninic acid assay kit (Thermo Fisher Scientific, Inc.) was used to measure protein concentrations. Proteins (40 $\mu \mathrm{g} / \mathrm{lane})$ were separated via $12 \%$ SDS-PAGE gel and electrically transferred onto PVDF membranes. After blocking with 5\% skim milk at room temperature for $1.5 \mathrm{~h}$, the membranes were incubated at $4^{\circ} \mathrm{C}$ overnight with primary antibodies targeted against: Smad7 (1:1,000; cat. no. ab227309; Abcam), $\alpha$-smooth muscle actin ( $\alpha$-SMA; $1: 1,000$; cat. no. ab32575; Abcam), type I collagen (Col I; 1:1,000; cat. no. ab34710; Abcam), type III collagen (Col III; 1:1,000; cat. no. ab7778; Abcam) and GAPDH (1:1,000; cat. no. ab9485; Abcam). Subsequently, the membranes were incubated with an anti-rabbit horseradish peroxidase-conjugated IgG secondary antibody (cat. no. 7074; 1:2,000; Cell Signaling Technology, Inc.) at room temperature for $2 \mathrm{~h}$. Protein bands 

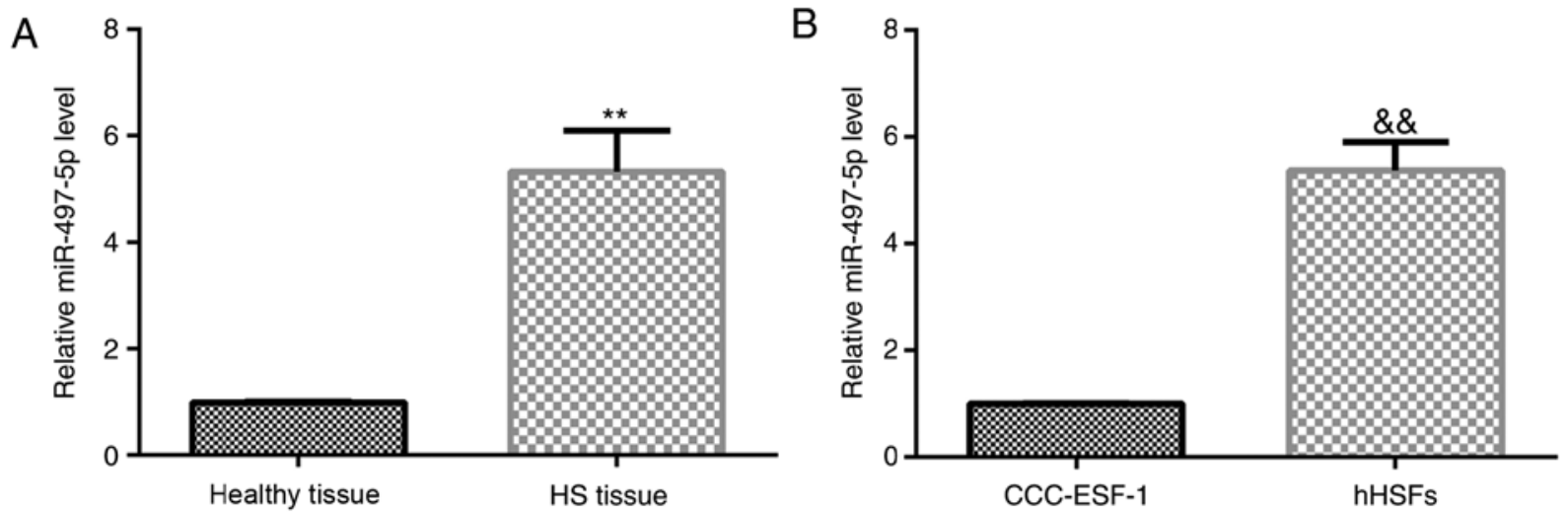

Figure 1. miR-497-5p expression is upregulated in HS tissues and cells. Relative miR-497-5p expression levels in (A) HS tissue, healthy tissue, (B) CCC-ESF-1 cells and hHSFs. " $\mathrm{P}<0.01$ vs. healthy tissues; \&\& $\mathrm{P}<0.01$ vs. CCC-ESF-1. miR, micro RNA; HS, hypertrophic scar; hHSFs, human hyperplastic scar fibroblasts.
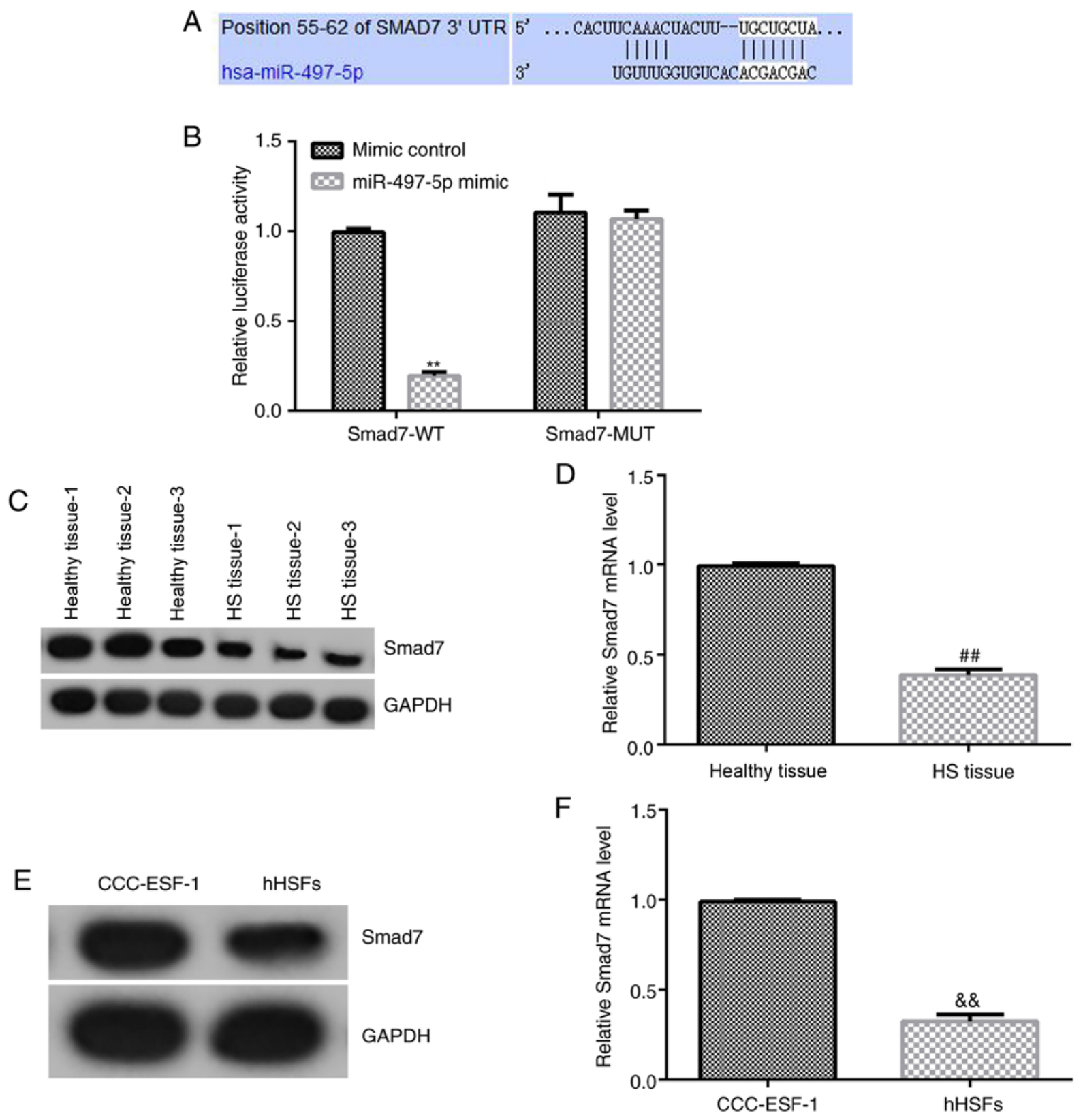

Figure 2. Smad7 is a target gene of miR-497-5p. (A) The binding sites between miR-497-5p and the 3'-UTR of Smad7 were predicted using TargetScan. (B) Luciferase activity was measured by performing a dual-luciferase reporter assay. Smad7 mRNA and protein expression levels in (C and D) HS tissues, healthy tissues, (E and F) CCC-ESF-1 cells and hHSFs. ${ }^{* *} \mathrm{P}<0.01$ vs. mimic control; ${ }^{* \#} \mathrm{P}<0.01$ vs. healthy tissues; ${ }^{\text {\&\&}} \mathrm{P}<0.01$ vs. CCC-ESF-1. miR, micro RNA; 3'-UTR, 3'-untranslated region; HS, hypertrophic scar; WT, wild-type; MUT, mutant. 


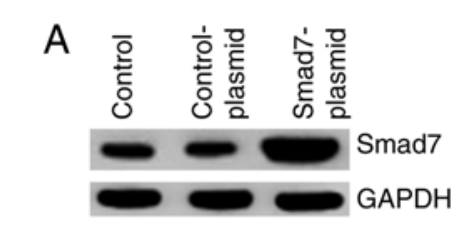

B
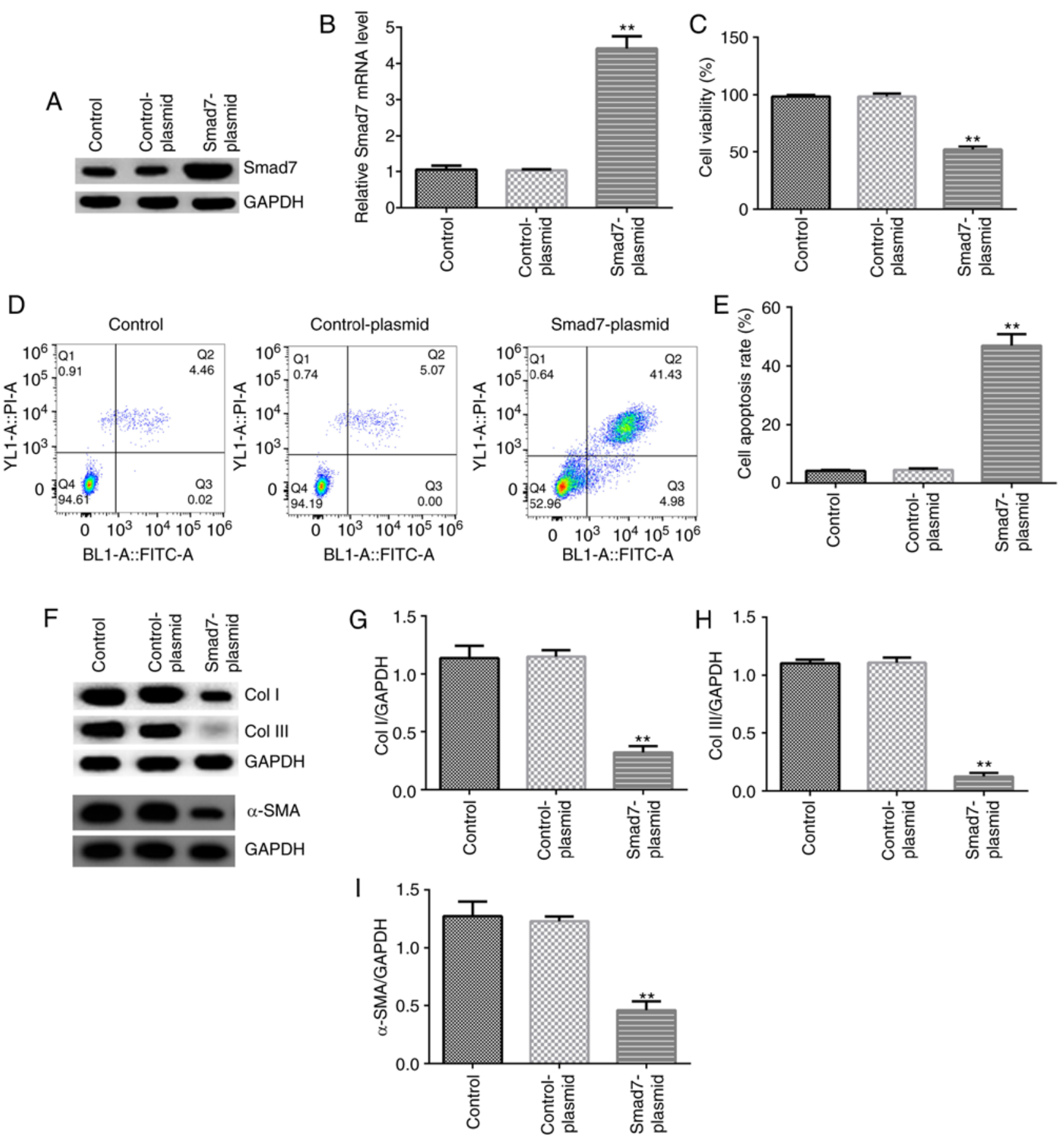

Figure 3. Effect of Smad7 on hHSFs. hHSFs were transfected with control-plasmid or Smad7-plasmid for $48 \mathrm{~h}$. (A) Protein and (B) mRNA expression levels of Smad7. (C) Cell viability was assessed by performing the Cell Counting Kit-8 assay. Cell apoptosis was (D) determined by flow cytometry and (E) quantified. Protein expression levels were (F) determined by western blotting and semi-quantified for (G) Col I, (H) Col III and (I) $\alpha$-SMA. * ${ }^{*}<<0.01$ vs. control-plasmid. hHSFs, human hyperplastic scar fibroblasts; Col I, type I collagen; Col III, type III collagen; $\alpha$-SMA, $\alpha$-smooth muscle actin.

were visualized using an enhanced chemiluminescence detection system (Thermo Fisher Scientific, Inc.). ImageJ version 2.0 software (National Institutes of Health) was used to quantify band intensities.

Statistical analysis. Data are presented as the mean \pm SD of three independent experiments. Statistical analyses were performed using SPSS software (version 18.0; SPSS, Inc.). Comparisons between two groups were analyzed using the Student's t-test. Comparisons among multiple groups were analyzed using one-way ANOVA followed by Tukey's post hoc test. $\mathrm{P}<0.05$ was considered to indicate a statistically significant difference.

\section{Results}

miR-497-5p is upregulated in HS tissues and hHSFs. miR-497-5p expression levels in 22 HS tissues, 22 healthy control skin tissues, human embryonic skin fibroblasts CCC-ESF-1 and hHSFs were determined via RT-qPCR. The results indicated that the expression levels of miR-497-5p were significantly upregulated in HS tissues compared with healthy 


\section{A}
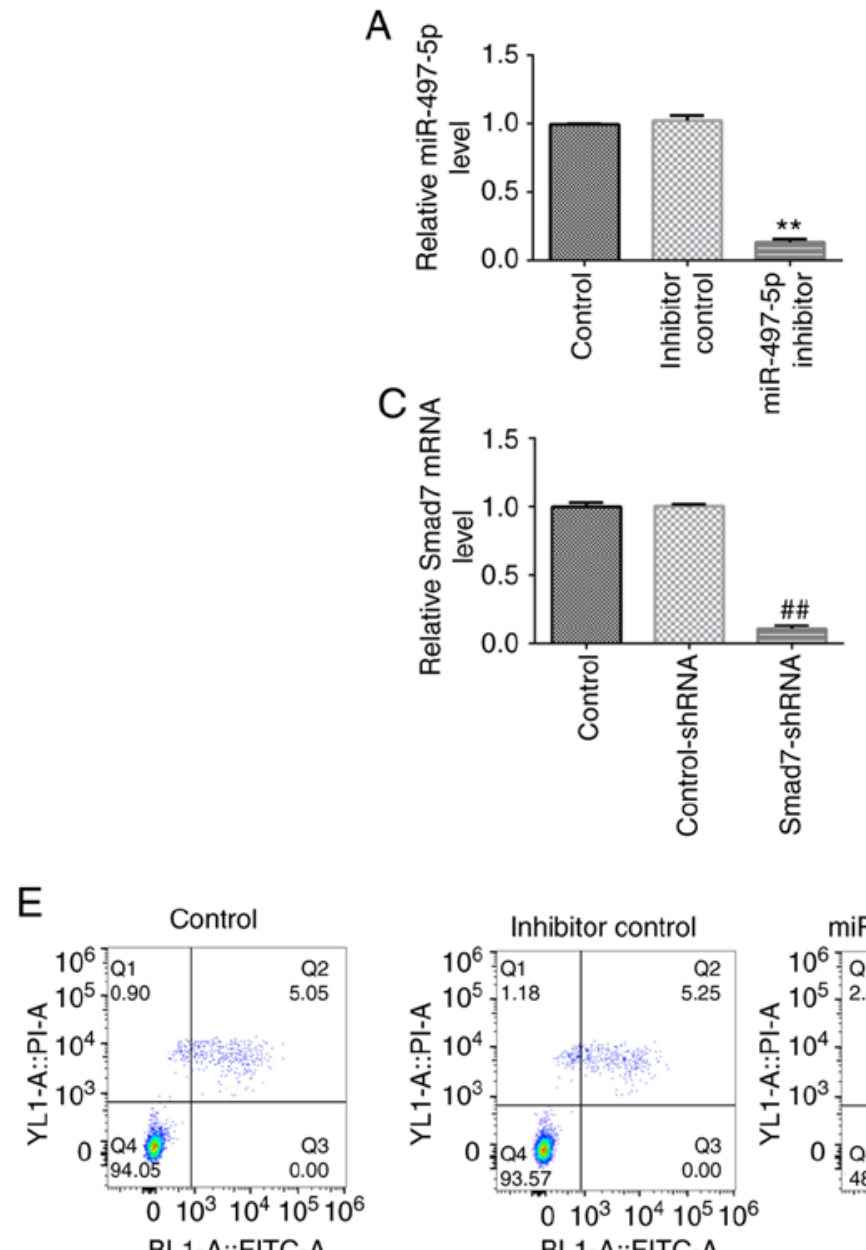

BL1-A::FITC-A

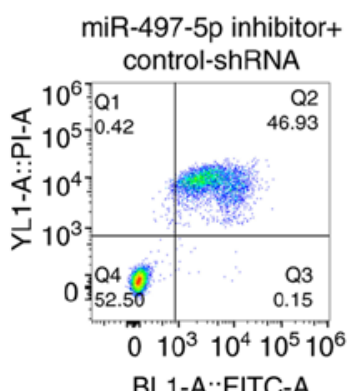

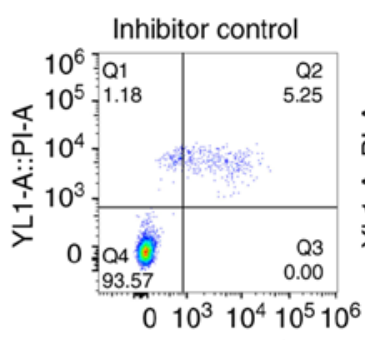

BL1-A::FITC-A

miR-497-5p inhibitor+

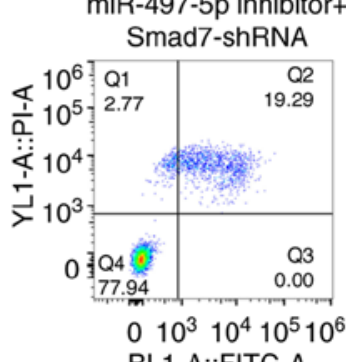

B
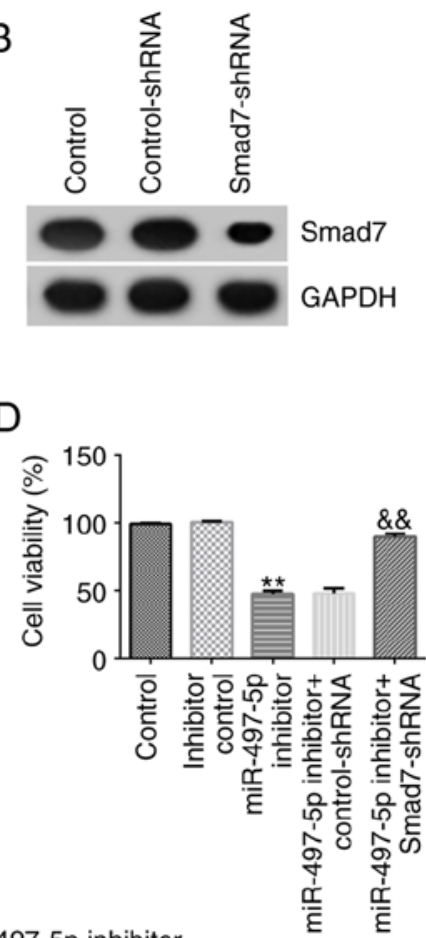

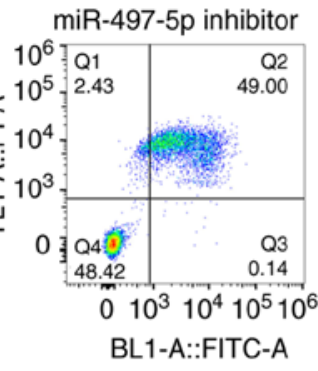

$\mathrm{F}$

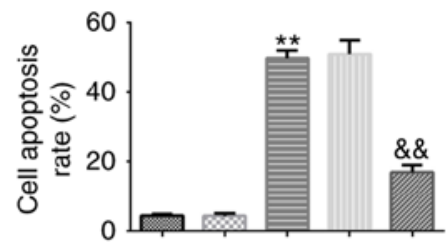

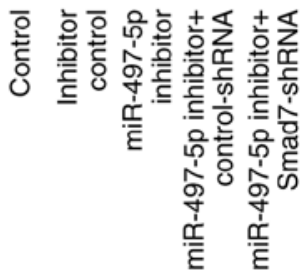

Figure 4. Effect of miR-497-5p inhibitor on hHSF viability and apoptosis. (A) miR-497-5p expression levels in hHSFs transfected with inhibitor control or miR-497-5p inhibitor for $48 \mathrm{~h}$. (B) Protein and (C) mRNA expression levels of Smad7 in hHSFs transfected with control-shRNA or Smad7-shRNA for $48 \mathrm{~h}$. hHSFs were transfected with inhibitor control, miR-497-5p inhibitor, miR-497-5p inhibitor + control-shRNA or miR-497-5p inhibitor + Smad7-shRNA for $48 \mathrm{~h}$. (D) The Cell Counting Kit-8 assay was performed to determine hHSF viability. Cell apoptosis was (E) determined by flow cytometry and (F) quantified. ${ }^{* *} \mathrm{P}<0.01$ vs. inhibitor control; ${ }^{\# /} \mathrm{P}<0.01$ vs. control-shRNA; ${ }^{\& \&} \mathrm{P}<0.01$ vs. miR-497-5p inhibitor + control-shRNA. miR, micro RNA; hHSF, human hyperplastic scar fibroblast; shRNA, short hairpin RNA.

control skin tissues (Fig. 1A). Similarly, the expression level of miR-497-5p in hHSFs was significantly higher compared with CCC-ESF-1 cells (Fig. 1B). The results indicated that miR-497-5p may be involved in the development of HS.

Smad7 is a target gene of miR-497-5p. TargetScan was used to predict the potential targets of miR-497-5p, which indicated a binding site between miR-497-5p and the 3'UTR of Smad7 mRNA (Fig. 2A). Subsequently, a dual-luciferase reporter assay was conducted to assess the binding site between miR-497-5p and the 3'UTR of Smad7
(Fig. 2B). Compared with cells co-transfected with mimic control and Smad7-WT, the luciferase activity of cells co-transfected with miR-497-5p mimic and Smad7-WT was significantly reduced. By contrast, there was no significant difference between the luciferase activity of cells co-transfected with mimic control and Smad7-MUT, and cells co-transfected with miR-497-5p mimic and Smad7-MUT. The results indicated that Smad7 may be a direct target gene of miR-497-5p.

Furthermore, compared with healthy control skin tissues and CCC-ESF-1 cells, the mRNA and protein expression levels 
A

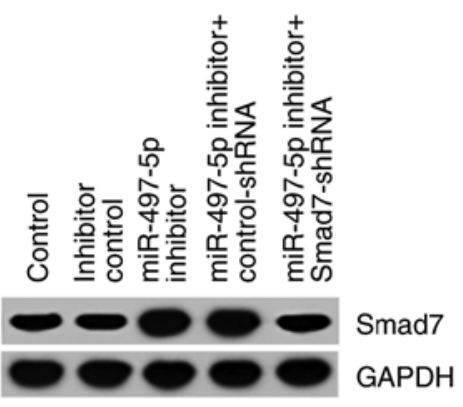

B

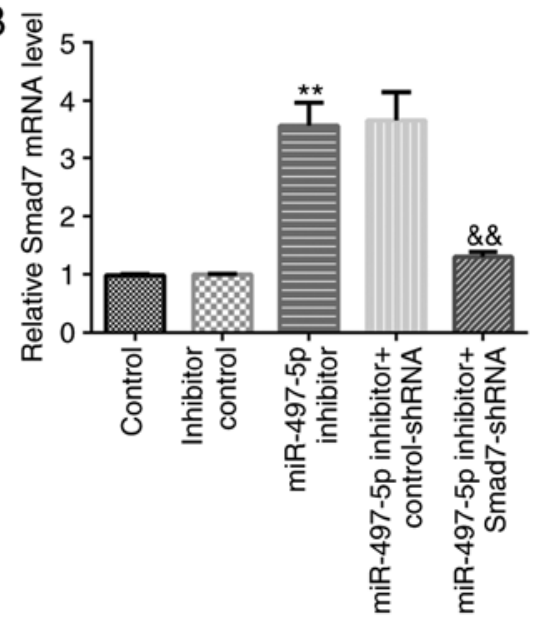

E 1.5
C

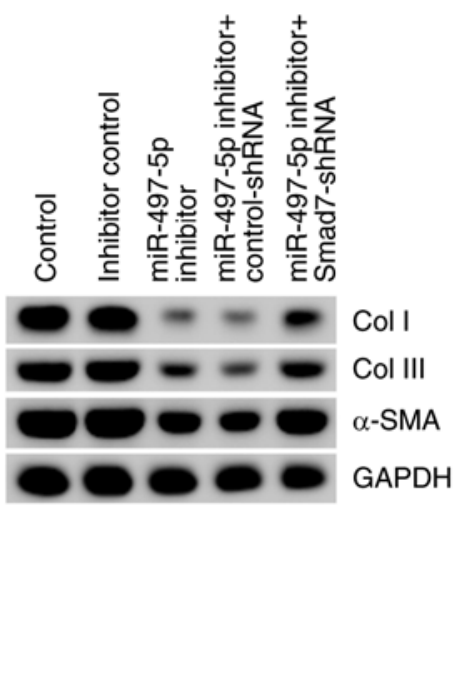

$\mathrm{F}$

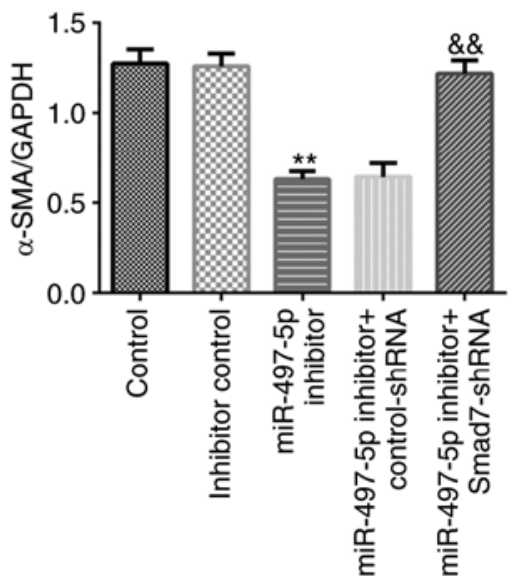

Figure 5. Effect of miR-497-5p inhibitor on Smad7, Col I, Col III and $\alpha$-SMA expression in hHSFs. hHSFs were transfected with control-shRNA, Smad7-shRNA, inhibitor control, miR-497-5p inhibitor, miR-497-5p inhibitor + control-shRNA or miR-497-5p inhibitor + Smad7-shRNA for 48 h. (A) Protein and (B) mRNA expression levels of Smad7. Protein expression levels were (C) determined by western blotting and semi-quantified for (D) Col I, (E) Col III and (F) $\alpha$-SMA. ${ }^{* *} \mathrm{P}<0.01$ vs. inhibitor control ${ }^{\&} \mathrm{P}<0.01$ vs. miR-497-5p inhibitor + control-shRNA. miR, microRNA; Col I, type I collagen; Col III, type III collagen; $\alpha$-SMA, $\alpha$-smooth muscle actin; hHSFs, human hyperplastic scar fibroblasts; shRNA, short hairpin RNA.

of Smad7 were significantly downregulated in HS tissues and hHSFs, respectively (Fig. 2C-F).

Effect of Smad7 on hHSFs. The effect of Smad7 was investigated in hHSFs. hHSFs were transfected with control-plasmid or Smad7-plasmid for $48 \mathrm{~h}$. Compared with the control-plasmid group, Smad7-plasmid significantly increased Smad7 mRNA and protein expression levels in hHSFs (Fig. 3A and B). In addition, compared with the control-plasmid group, Smad7-plasmid significantly decreased cell viability, enhanced apoptosis, and inhibited the protein expression of Col I, Col III and $\alpha$-SMA in hHSFs (Fig. 3C-I).

Effect of miR-497-5p on hHSF viability and apoptosis. The effect of miR-497-5p on hHSF viability and apoptosis was analyzed. Compared with the inhibitor control group, miR-497-5p inhibitor significantly inhibited miR-497-5p expression in hHSFs (Fig. 4A). Smad7-shRNA notably decreased the mRNA and protein expression levels of Smad7 in hHSFs compared with the control-shRNA group (Fig. 4B and C). miR-497-5p inhibitor-mediated reduction in
hHSF viability and induction of apoptosis were reversed by co-transfection with Smad7-shRNA (Fig. 4D-F).

Effect of miR-497-5p on the protein expression of Smad7, Col I, Col III and $\alpha$-SMA in hHSFs. The effect of miR-497-5p on extracellular matrix deposition in hHSFs was investigated. At $48 \mathrm{~h}$ post-transfection, the expression of Smad7, Col I, Col III and $\alpha$-SMA in hHSFs was assessed. The results indicated that miR-497-5p inhibitor markedly increased the mRNA and protein expression levels of Smad7 in hHSFs compared with the inhibitor control group (Fig. 5A and B), which was reversed by co-transfection with Smad7-shRNA. Moreover, the results indicated that miR-497-5p inhibitor significantly decreased the protein expression levels of Col I, Col III and $\alpha$-SMA in hHSFs compared with the inhibitor control group, which was reversed by co-transfection with Smad7-shRNA (Fig. 5C-F).

\section{Discussion}

The present study demonstrated that miR-497-5p was upregulated in HS tissues and hHSFs compared with healthy control 
skin tissues and CCC-ESF-1 cells, respectively. Smad7 was a direct target of miR-497-5p, and was downregulated in HS tissues and hHSFs compared with healthy control skin tissues and CCC-ESF-1 cells, respectively. miR-497-5p downregulation significantly inhibited ECM deposition, inhibited hHSF proliferation and induced cell apoptosis compared with the inhibitor-control group, which were all reversed by Smad7 downregulation. Collectively, the results of the present study may aid with the development of novel therapeutic strategies for HS.

To date, HS has been the focus of research on trauma and plastic surgery (30). miRNAs have been identified to serve critical roles in the progression of skin wound repair and skin diseases $(31,32)$. Research on miRNAs in HS has attracted increasing attention (33-38). miR-497-5p, which has been reported to serve critical roles in the regulation of cell proliferation and apoptosis (17-21), was investigated in the present study.

The level of miR-497-5p was detected in HS tissues, healthy control skin tissues, human embryonic skin fibroblasts CCC-ESF-1 and hHSFs via RT-qPCR. The results suggested that miR-497-5p was highly expressed in HS tissues and hHSFs compared with healthy control skin tissues and CCC-ESF-1 cells, respectively, and Smad7 was identified as a direct target of miR-497-5p. Consistent with a previous study (39), the present study indicated that Smad7 was downregulated in HS tissues and hHSFs compared with healthy control skin tissues and CCC-ESF-1 cells, respectively. The results suggested that miR-497-5p might participate in HS formation by regulating the expression of Smad7. HS is characterized by abnormal fibroblast proliferation, and collagen and ECM deposition (4,5). Zhang et al (35) reported that miR-137 inhibits HS fibroblast proliferation and metastasis via targeting pleiotrophin. Wu et al (36) suggested that miR-155 inhibits the formation of HSs by inhibiting HS fibroblast proliferation and collagen expression by targeting hypoxia inducible factor- $1 \alpha$ via the PI3K/AKT signaling pathway. Zhou et al (37) indicated that miR-519d inhibits proliferation and induces apoptosis of human HS fibroblasts, serving a critical role in HS formation. Moreover, previous studies have reported that Smad7 serves a key role in the regulation of fibroblast growth and ECM accumulation (26-28). Therefore, the present study investigated the effect of Smad7 on ECM secretion, and hHSF proliferation and apoptosis. The results indicated that Smad7 upregulation significantly inhibited cell viability, induced cell apoptosis, and decreased the protein expression levels of Col I, Col III and $\alpha$-SMA in hHSFs compared with the control-plasmid group. miRNAs work by negatively regulating the expression of target genes $(40,41)$. Using bioinformatics software, Smad7 was identified as a potential target gene of miR-497-5p in the present study. Therefore, it was hypothesized that miR-497-5p may regulate scar fibroblast proliferation and ECM production by regulating the expression of Smad7, thereby serving a role in HS formation. As expected, the results of the present study indicated that miR-497-5p inhibition decreased cell viability, induced apoptosis, and decreased the protein expression levels of Col I, Col III and $\alpha$-SMA in hHSFs compared with the inhibitor control group. Notably, miR-497-5p knockdown-mediated effects on hHSFs were eliminated by Smad7 downregulation. However, in the present study, only the CCK-8 assay was performed to assess the effect of miR-497-5p/Smad7 on hHSF viability, which was a limitation. Future studies should perform EdU staining to investigate the effect of miR-497-5p/Smad7 on hHSF proliferation.

In summary, the present study indicated that miR-497-5p was upregulated in HS. miR-497-5p downregulation inhibited HS formation by inhibiting ECM deposition, preventing hHSF proliferation at least partly by targeting Smad7. Therefore, the miR-497-5p/Smad7 axis may serve as a promising therapeutic target for the treatment of HS. However, the present study was only a preliminary study of the effect of miR-497-5p on hHSFs and further investigation is required. For example, the effects of miR-497-5p/Smad7 on hHSF migration and the role of the miR-497-5p/Smad7 axis in HSs in vivo require further investigation. Moreover, whether there is a correlation between the expression of $\mathrm{miR}-497-5 \mathrm{p} / \mathrm{Smad} 7$ and the clinicopathological parameters of patients with HS should be investigated in the future.

\section{Acknowledgements}

Not applicable.

\section{Funding}

No funding was received.

\section{Availability of data and materials}

The datasets used and/or analyzed during the current study are available from the corresponding author on reasonable request.

\section{Authors' contributions}

ZL designed the study, collected, analyzed and interpreted the data and prepared the manuscript. PW, JZ and DZ collected the data and performed statistical analysis. All authors read and approved the final manuscript.

\section{Ethics approval and consent to participate}

The present study was approved by the Ethics Committee of Shanghai Meizhizhen Medical Cosmetology Clinic. Written informed consent was obtained from the patients and all patients approved the use of their samples in the present study.

\section{Patient consent for publication}

Not applicable.

\section{Competing interests}

The authors declare that they have no competing interests.

\section{References}

1. Gabriel V: Hypertrophic scar. Phys Med Rehabil Clin N Am 22: 301-310, 2011.

2. Fan SQ, Cai JL, Qin LY, Wang ZH, Liu ZZ and Sun ML: Effect of heparin on production of transforming growth factor (TGF)-betal and TGF-betal mRNA expression by human normal skin and hyperplastic scar fibroblasts. Ann Plast Surg 60: 299-305, 2008. 
3. Gauglitz GG, Korting HC, Pavicic T, Ruzicka T and Jeschke MG: Hypertrophic scarring and keloids: Pathomechanisms and current and emerging treatment strategies. Mol Med 17: 113-125, 2011.

4. Sidgwick GP, Iqbal SA and Bayat A: Altered expression of hyaluronan synthase and hyaluronidase mRNA may affect hyaluronic acid distribution in keloid disease compared with normal skin. Exp Dermatol 22: 377-379, 2013.

5. Syed F, Ahmadi E, Iqbal SA, Singh S, McGrouther DA and Bayat A: Fibroblasts from the growing margin of keloid scars produce higher levels of collagen I and III compared with intralesional and extralesional sites: Clinical implications for lesional site-directed therapy. Br J Dermatol 164: 83-96, 2011.

6. Zuccaro J, Ziolkowski N and Fish J: A systematic review of the effectiveness of laser therapy for hypertrophic burn scars. Clin Plast Surg 44: 767-779, 2017.

7. Xu X, Lai L, Zhang X, Chen J, Chen J, Wang F, Zheng J and Chen M: Autologous chyle fat grafting for the treatment of hypertrophic scars and scar-related conditions. Stem Cell Res Ther 9: 64, 2018

8. Bartel DP: MicroRNAs: Genomics, biogenesis, mechanism, and function. Cell 116: 281-297, 2004.

9. Ebert MS and Sharp PA: Roles for microRNAs in conferring robustness to biological processes. Cell 149: 515-524, 2012.

10. Rogers K and Chen X: Biogenesis, turnover, and mode of action of plant microRNAs. Plant Cell 25: 2383-2399, 2013.

11. Hayes J, Peruzzi PP and Lawler S: MicroRNAs in cancer: Biomarkers, functions and therapy. Trends Mol Med 20: 460-469, 2014.

12. Wojtas B, Ferraz C, Stokowy T, Hauptmann S, Lange D, Dralle H, Musholt T, Jarzab B, Paschke R and Eszlinger M: Differential miRNA expression defines migration and reduced apoptosis in follicular thyroid carcinomas. Mol Cell Endocrinol 388: 1-9, 2014.

13. Mu S, Kang B, Zeng W, Sun Y and Yang F: MicroRNA-143-3p inhibits hyperplastic scar formation by targeting connective tissue growth factor $\mathrm{CTGF} / \mathrm{CCN} 2$ via the Akt/mTOR pathway. Mol Cell Biochem 416: 99-108, 2016.

14. Hao XZ and Fan HM: Identification of miRNAs as atherosclerosis biomarkers and functional role of miR-126 in atherosclerosis progression through MAPK signalling pathway. Eur Rev Med Pharmacol Sci 21: 2725-2733, 2017

15. Bi S, Chai L, Yuan X, Cao C and Li S: MicroRNA-98 inhibits the cell proliferation of human hypertrophic scar fibroblasts via targeting Col1A1. Biol Res 50: 22, 2017.

16. Zhu HY, Li C, Bai WD, Su LL, Liu JQ, Li Y, Shi JH, Cai WX, Bai XZ, Jia YH, et al: MicroRNA-21 regulates hTERT via PTEN in hypertrophic scar fibroblasts. PLoS One 9: e97114, 2014.

17. Qu F, Ye J, Pan X, Wang J, Gan S, Chu C, Chu J, Zhang X, Liu M, $\mathrm{He} \mathrm{H}$ and Cui X: MicroRNA-497-5p down-regulation increases PD-L1 expression in clear cell renal cell carcinoma. J Drug Target 27: 67-74, 2019.

18. Chai L, Kang XJ, Sun ZZ, Zeng MF, Yu SR, Ding Y, Liang JQ, Li TT and Zhao J: MiR-497-5p, miR-195-5p and miR-455-3p function as tumor suppressors by targeting hTERT in melanoma A375 cells. Cancer Manag Res 10: 989-1003, 2018.

19. Sun Z, Li A, Yu Z, Li X, Guo X and Chen R: MicroRNA-497-5p suppresses tumor cell growth of osteosarcoma by targeting ADP ribosylation factor-like protein 2. Cancer Biother Radiopharm 32: 371-378, 2017.

20. Chen Y, Kuang D, Zhao X, Chen D, Wang X, Yang Q, Wan J, Zhu Y, Wang Y, Zhang S, et al: miR-497-5p inhibits cell proliferation and invasion by targeting $\mathrm{KCa} 3.1$ in angiosarcoma. Oncotarget 7: 58148-58161, 2016.

21. Chen X, Shi C, Wang C, Liu W, Chu Y, Xiang Z, Hu K, Dong P and Han X: The role of miR-497-5p in myofibroblast differentiation of LR-MSCs and pulmonary fibrogenesis. Sci Rep 7: 40958, 2017.

22. Nakao A, Afrakhte M, Morén A, Nakayama T, Christian JL, Heuchel R, Itoh S, Kawabata M, Heldin NE, Heldin CH and ten Dijke P: Identification of Smad7, a TGFbeta-inducible antagonist of TGF-beta signalling. Nature 389: 631-635, 1997.
23. Kavsak P, Rasmussen RK, Causing CG, Bonni S, Zhu H, Thomsen GH and Wrana JL: Smad7 binds to Smurf2 to form an E3 ubiquitin ligase that targets the TGF beta receptor for degradation. Mol Cell 6: 1365-1375, 2000

24. Yeung ML, Yao Y, Jia L, Chan JFW, Chan KH, Cheung KF, Chen H, Poon VKM, Tsang AKL, To KKW, et al: MERS coronavirus induces apoptosis in kidney and lung by upregulating Smad7 and FGF2. Nat Microbiol 1: 16004, 2016.

25. Liu J, Kong D, Qiu J, Xie Y, Lu Z, Zhou C, Liu X, Zhang R and Wang Y: Praziquantel ameliorates $\mathrm{CCl}_{4}$-induced liver fibrosis in mice by inhibiting TGF- $\beta /$ Smad signalling via up-regulating Smad7 in hepatic stellate cells. Br J Pharmacol 176: 4666-4680, 2019.

26. Zhou R, Wang C, Wen $\mathrm{C}$ and Wang D: miR-21 promotes collagen production in keloid via Smad7. Burns 43: 555-561, 2017.

27. Chen Z, Liu Y, Xiao M, Xiao H, Qiu W and Zhao Y: Effects of over-expressing smad7 gene on keloid fibroblasts. Zhongguo Xiu Fu Chong Jian Wai Ke Za Zhi 30: 871-875, 2016 (In Chinese).

28. Tao J, Wang J, Li C, Wang W, Yu H, Liu J, Kong X and Chen Y: MiR-216a accelerates proliferation and fibrogenesis via targeting PTEN and SMAD7 in human cardiac fibroblasts. Cardiovasc Diagn Ther 9: 535-544, 2019.

29. Livak KJ and Schmittgen TD: Analysis of relative gene expression data using real-time quantitative PCR and the 2(-Delta Delta C(T)) method. Methods 25: 402-408, 2001

30. Lee HJ and Jang YJ: Recent understandings of biology, prophylaxis and treatment strategies for hypertrophic scars and keloids. Int J Mol Sci 19: 711, 2018.

31. Aberdam D, Candi E, Knight RK and Melino G: miRNAs, 'sternness' and skin. Trends Biochem Sci 33: 583-591, 2008.

32. Botchkareva NV: MicroRNA/mRNA regulatory networks in the control of skin development and regeneration. Cell Cycle 11: 468-474, 2012

33. Chen L, Li J, Li Q, Yan H, Zhou B, Gao Y and Li J: Non-coding RNAs: The new insight on hypertrophic scar. J Cell Biochem 118: 1965-1968, 2017.

34. Tsai CH and Ogawa R: Keloid research: Current status and future directions. Scars Burn Heal 5: 2059513119868659, 2019.

35. Zhang Q, Guo B, Hui Q, Chang P and Tao K: miR-137 inhibits proliferation and metastasis of hypertrophic scar fibroblasts via targeting pleiotrophin. Cell Physiol Biochem 49: 985-995, 2018.

36. Wu X, Li J, Yang X, Bai X, Shi J, Gao J, Li Y, Han S, Zhang Y, Han F, et al: miR-155 inhibits the formation of hypertrophic scar fibroblasts by targeting HIF-1 $\alpha$ via PI3K/AKT pathway. J Mol Histol 49: 377-387, 2018.

37. Zhou X, Xie Y, Xiao H, Deng X, Wang Y, Jiang L, Liu C and Zhou R: MicroRNA-519d inhibits proliferation and induces apoptosis of human hypertrophic scar fibroblasts through targeting Sirtuin 7. Biomed Pharmacother 100: 184-190, 2018.

38. Wang X, Zhang Y, Jiang BH, Zhang Q, Zhou RP, Zhang L and Wang C: Study on the role of Hsa-miR-31-5p in hypertrophic scar formation and the mechanism. Exp Cell Res 361: 201-209, 2017.

39. Zunwen L, Shizhen Z, Dewu L, Yungui M and Pu N: Effect of tetrandrine on the TGF- $\beta$-induced smad signal transduction pathway in human hypertrophic scar fibroblasts in vitro. Burns 38: 404-413, 2012.

40. Liu B, Li J and Cairns MJ: Identifying miRNAs, targets and functions. Brief Bioinform 15: 1-19, 2014.

41. Lu TX and Rothenberg ME: MicroRNA. J Allergy Clin Immunol 141: 1202-1207, 2018.

This work is licensed under a Creative Commons Attribution-NonCommercial-NoDerivatives 4.0 International (CC BY-NC-ND 4.0) License. 